\title{
Plasma receptor interacting protein kinase-3 levels are associated with acute respiratory distress syndrome in sepsis and trauma: a cohort study
}

Michael G. S. Shashaty ${ }^{1,2,3^{*+}}$ (D), John P. Reilly ${ }^{1,3+}$, Hilary E. Faust ${ }^{1,3}$, Caitlin M. Forker ${ }^{1}$, Caroline A. G. Ittner ${ }^{1}$, Peggy X. Zhang ${ }^{1}$, Meghan J. Hotz', David Fitzgerald ${ }^{1}$, Wei Yang ${ }^{2}$, Brian J. Anderson ${ }^{1,3}$, Daniel N. Holena ${ }^{4}$, Paul N. Lanken', Jason D. Christie ${ }^{1,2,3}$, Nuala J. Meyer ${ }^{1,3}$ and Nilam S. Mangalmurti ${ }^{1,3}$

\begin{abstract}
Background: Necroptosis, a form of programmed cell death mediated by receptor interacting serine/threonineprotein kinase-3 (RIPK3), is implicated in murine models of acute respiratory distress syndrome (ARDS). We hypothesized that plasma RIPK3 concentrations in sepsis and trauma would be associated with ARDS development and that plasma RIPK3 would reflect changes in lung tissue RIPK3 in a murine model of systemic inflammation.

Methods: We utilized prospective cohort studies of critically ill sepsis $(n=120)$ and trauma $(n=180)$ patients and measured plasma RIPK3 at presentation and $48 \mathrm{~h}$. Patients were followed for 6 days for ARDS by the Berlin definition. We used multivariable logistic regression to determine the association of plasma RIPK3 with ARDS in each cohort, adjusting for confounders. In mice, we determined whether plasma and lung tissue RIPK3 levels rise concomitantly $4 \mathrm{~h}$ after injection with lipopolysaccharide and ZVAD-FMK, an apoptosis inhibitor.
\end{abstract}

Results: The change in plasma RIPK3 from presentation to $48 \mathrm{~h}$ ( $\triangle \mathrm{RIPK} 3$ ) was associated with ARDS in sepsis (OR 1.30, 95\% Cl 1.03-1.63, per $1 / 2$ standard deviation) and trauma (OR 1.79, 95\% Cl 1.33-2.40). This association was not evident for presentation RIPK3 levels. Secondary analyses showed similar findings for the association of $\triangle R I P K 3$ with acute kidney injury and 30-day mortality. Mice injected with lipopolysaccharide and ZVAD-FMK had significantly higher plasma $(p<0.001)$ and lung $(p=0.005)$ RIPK3 than control mice.

Conclusions: The change in plasma RIPK3 from presentation to $48 \mathrm{~h}$ in both sepsis and trauma patients is independently associated with ARDS, and plasma RIPK3 may reflect RIPK3 activity in lung tissue.

Keywords: Necroptosis, Sepsis, Trauma, Acute respiratory distress syndrome, Acute kidney injury

\section{Background}

Critical illness precipitated by sepsis or trauma is characterized by a dysregulated immune response that may result in organ dysfunction and consequent death or long-term morbidity [1-4]. Despite strides in early management strategies

\footnotetext{
* Correspondence: shashatm@pennmedicine.upenn.edu

${ }^{\dagger}$ Michael G. S. Shashaty and John P. Reilly contributed equally to this work.

'Pulmonary, Allergy, and Critical Care Division, Perelman School of Medicine, University of Pennsylvania, 5039 W Gates Building, 3600 Spruce Street,

Philadelphia, PA 19104, USA

${ }^{2}$ Center for Clinical Epidemiology and Biostatistics, Perelman School of

Medicine, University of Pennsylvania, Pennsylvania, USA

Full list of author information is available at the end of the article
}

$[5,6]$, organ dysfunction syndromes such as the acute respiratory distress syndrome (ARDS) often complicate the early clinical trajectory of these patients and pose a significant barrier to improving outcomes. Disappointing results of trials for immune-targeted and other pharmacologic therapies have prompted interest in better understanding clinically relevant molecular pathways in ARDS through human translational study [7].

Necroptosis, a caspase-independent form of regulated cellular necrosis mediated by receptor interacting serine/ threonine-protein kinase-3 (RIPK3) and mixed lineage kinase domain-like protein (MLKL) $[8,9]$, has recently

(c) The Author(s). 2019 Open Access This article is distributed under the terms of the Creative Commons Attribution 4.0 International License (http://creativecommons.org/licenses/by/4.0/), which permits unrestricted use, distribution, and 
been implicated as a key cell death modality in tissue and animal models of organ injury [10-13]. Studies by our group and others have shown that lipopolysaccharide- and red blood cell-induced lung injury is attenuated by inhibition of necroptosis $[10,13]$. This form of cell death could have particular relevance to ARDS. Unlike apoptosis, necroptosis results in plasma membrane rupture and release of damage-associated molecular patterns (DAMPs) that drive tissue injury; distinct from conventional descriptions of necrosis, necroptosis induction and execution is highly regulated $[9,14-18]$. Therefore, necroptosis may represent a novel potential therapeutic target for prevention or treatment of acute organ dysfunction.

Few studies to date have investigated necroptosis activation in critically ill patients. After finding that RIPK3 was released by cultured lung endothelial cells undergoing red blood cell-induced necroptosis, we reported an association of plasma RIPK3 with RBC transfusions and mortality in 37 sepsis patients [13]. We subsequently showed that plasma RIPK3 measured $48 \mathrm{~h}$ after presentation was associated with $\mathrm{RBC}$ transfusions and acute kidney injury (AKI) in 80 trauma patients [19]. Recent reports in medical ICU populations described higher plasma RIPK3 levels in mechanically ventilated and non-surviving patients $[20,21]$. These studies provided limited information on the relationship of plasma RIPK3 with ARDS. Further, it remains unclear whether plasma RIPK3 is an accurate marker of expression of RIPK3 or execution of necroptosis in injured organs.

We sought to address these knowledge gaps using cohorts of critically ill sepsis and trauma patients as well as in vivo animal experiments. We hypothesized that plasma RIPK3 levels would be associated with ARDS in both sepsis and trauma populations, independent of patient-level characteristics. We also hypothesized that in a mouse model of systemic inflammation, plasma RIPK3 levels would correlate with lung tissue expression of the necroptosis mediators RIPK3 and MLKL. Secondarily, we sought to build on our prior reports $[13,19]$ by determining the association of plasma RIPK3 levels with AKI and mortality in larger cohorts of sepsis and trauma patients and by determining patient characteristics associated with plasma RIPK3. We studied these populations based on two considerations: first, sepsis and trauma are common critical illness syndromes with high rates of organ dysfunction; and second, sepsis and trauma are both characterized by a dysregulated immune response, ischemia-reperfusion injury, and treatment with blood product transfusions, all relevant for translation of existing pre-clinical studies of necroptosis $[12,13,16,18]$.

\section{Methods}

Detailed descriptions of human cohort and experimental animal studies, including the STROBE checklist, are in Additional file 1. Key methods are summarized here.

\section{Sepsis and trauma cohorts}

The Molecular Epidemiology of SepsiS in the Intensive care unit (MESSI) cohort and the PEnn TRauma Organ dysfunction Study (PETROS) are prospective cohort studies of critically ill sepsis and trauma patients, respectively, at the University of Pennsylvania [3, 22-24]. We included patients presenting to the emergency department and admitted to the medical ICU (MESSI, 2012-2014) or Penn Level I Trauma Center ICU (PETROS, 2012-2015) with plasma samples available at presentation and approximately $48 \mathrm{~h}$ later (Plasma collection, below). MESSI patients met American College of Chest Physicians/Society of Critical Care Medicine consensus criteria for severe sepsis or septic shock [25]. In PETROS, key exclusions were injury severity score (ISS) $<16$ or death within $24 \mathrm{~h}$ of admission. Both studies were approved by the University of Pennsylvania Institutional Review Board.

\section{Data collection and outcomes}

Clinical data were collected by medical record review. After prospective enrollment of patients, trained research personnel used REDCap-based electronic case report forms [26] to collect detailed medical history, physiologic variables, lab results, and treatment variables. We used the Berlin definition, with direct radiograph review by investigators, to identify incident ARDS and classify it as mild, moderate, or severe over the 6 days following presentation [27]. Patients were only considered to have ARDS if they were invasively mechanically ventilated at the time that they met ARDS criteria. We defined AKI by Acute Kidney Injury Network (AKIN) creatinine and renal replacement therapy (RRT) consensus criteria over the same time period [3, 28]. Mortality was determined at 30 days after admission.

\section{Plasma collection and RIPK3 measurement}

We utilized blood samples drawn for clinical purposes at presentation to the emergency department and approximately $48 \mathrm{~h}$ after presentation. Samples were centrifuged within $30 \mathrm{~min}$ and refrigerated at $4{ }^{\circ} \mathrm{C}$. Plasma aliquots were frozen within 12-48 h. We used enzyme-linked immunosorbent assay (Cusabio) to measure plasma RIPK3 concentrations [13, 19]. Plasma concentrations below the limit of detection $(15.6 \mathrm{pg} / \mathrm{ml})$ were set to $15.6 \mathrm{pg} / \mathrm{ml}$ for statistical analysis. Because our prior studies showed low RIPK3 levels at presentation in most trauma patients [19], and in order to account for RIPK3 release in the early stages of critical illness, we used the change in plasma RIPK3 from presentation to $48 \mathrm{~h}(\Delta \mathrm{RIPK} 3)$ for primary analyses. 


\section{Statistical analysis}

In primary analyses, by cohort (MESSI, PETROS), we tested unadjusted associations of patient characteristics with ARDS using Student's $t$, Wilcoxon rank-sum, $\chi^{2}$, or Fisher's exact tests. We used multivariable logistic regression models to test the associations of $\triangle$ RIPK3 with ARDS adjusted for confounders. To avoid overfitting, we limited explanatory variables to approximately one for every ten outcomes [29]. We pre-specified these variables by cohort (Additional file 1) based on previously described associations with ARDS or RIPK3 [13, 19, 30]. We used post-estimation marginal analysis to determine adjusted risk of ARDS across a range of plasma $\triangle$ RIPK 3 levels [31].

We repeated the primary $\triangle$ RIPK3-ARDS analyses stratified by several pre-specified patient characteristics (Additional file 1) and tested for interaction using likelihood ratio tests. In secondary analyses, we tested associations of $\triangle$ RIPK3 with AKI and mortality, associations of baseline characteristics with $\triangle \mathrm{RIPK} 3$, and differences in $\triangle \mathrm{RIPK} 3$ by organ dysfunction categories: ARDS alone, AKI alone, or both. Further secondary analysis details and sample size estimations are in Additional file 1. We used Stata/IC 13.1 (StataCorp, College Station, TX) and considered a twotailed $p<0.05$ significant for all analyses.

\section{Experimental animal studies}

All experimental procedures were performed on 812 -week-old female mice and conducted in accordance with the Institutional Animal Care and Use Committee at the University of Pennsylvania. Full details are in Additional file 1.

Mice were injected via tail vein with $10 \mathrm{mg} / \mathrm{kg}$ LPS (List Labs) as well as $10 \mathrm{mg} / \mathrm{kg}$ of the pan-caspase inhibitor ZVAD-FMK (BD Biosciences) in order to inhibit apoptosis and sensitize cells to necroptosis as previously described [16, 32-35]. Four hours following LPS-ZVAD administration, mice were sacrificed and both plasma and whole lungs were obtained for analysis. Plasma RIPK3 was measured using a murine ELISA kit (Cusabio). Lungs were homogenized and proteins were resolved by SDS-PAGE under reducing conditions. Immunoblotting was performed for RIPK3, MLKL, and phosphorylated MLKL normalized to $\beta$-actin. We determined differences in plasma and tissue RIPK3 between control and LPS groups using the Wilcoxon rank-sum test and tested the Spearman rank correlation between plasma and tissue RIPK3 (Stata/IC 13.1).

\section{Results}

\section{MESSI cohort}

From May 2012 to October 2014, 120 patients admitted from the emergency department were enrolled in the MESSI cohort and had plasma available at presentation and $48 \mathrm{~h}$. Cohort characteristics are shown in Table 1. Septic shock was present before ICU admission in $78 \%$, and the most common source of sepsis was pulmonary. ARDS developed in $44(37 \%)$ patients and AKI in 41 (34\%). Death at 30 days was more common in those with ARDS (55.6\% vs. $33.3 \%$ for no ARDS, $p=0.017$ ) or AKI (53.7\% vs. $37.1 \%$ for no AKI, $p=0.090)$. ARDS had a maximum severity of mild in $6(14 \%)$, moderate in 22 (50\%), and severe in $16(36 \%)$ cases.

\section{PETROS cohort}

From April 2012 to January 2015, 180 patients were enrolled in the PETROS cohort and had plasma available at presentation and $48 \mathrm{~h}$ (Table 1). The median ISS was 25 (19-29.5) and 137 (76.1\%) had blunt trauma mechanism. The median age was 20 years younger than in MESSI, 140 (79\%) were male, and blood product transfusions were far more common than in MESSI. ARDS occurred in 37 (20.6\%) patients and AKI in 53 (29.6\%). Mortality was much higher in patients who developed ARDS $(29.7 \%$ vs. $4.2 \%$ for no ARDS, $p<0.001)$ or AKI (17.0\% vs. $5.6 \%$ for no AKI, $p=0.014)$. ARDS had a maximum severity of mild in 7 (19\%), moderate in $21(57 \%)$, and severe in $9(24 \%)$ cases.

\section{Plasma RIPK3 and ARDS in human cohorts}

In both cohorts, patients who developed ARDS during the first 6 days had a significantly greater increase in plasma RIPK3 concentration from presentation to $48 \mathrm{~h}$ ( $\triangle$ RIPK3) than those who did not develop ARDS (Table 2). Findings were similar for plasma RIPK3 at 48 h. There was no association, however, between day 0 plasma RIPK3 levels and ARDS. Of note, 103 (57.2\%) trauma patients had day 0 levels below the limit of detection.

The association of $\triangle$ RIPK3 with ARDS remained significant in both cohorts in multivariable regression models adjusting for potential confounders (Table 3). Based on these models, Fig. 1 shows that the adjusted risk of ARDS increased from 30 to $>60 \%$ across the range of $\triangle$ RIPK3 in MESSI (1a) and from $<10 \%$ to $60 \%$ across the range of $\triangle$ RIPK3 in PETROS (1b).

In MESSI, the association of $\triangle$ RIPK 3 with ARDS was similar when patients were stratified by infection source (adjusted OR 1.31 (95\% CI 0.88-1.97) for pulmonary $(n=51) ; 1.30(0.98-1.72)$ for non-pulmonary $(n=69)$; interaction $p=0.844$ ) and did not differ significantly by shock (adjusted OR $1.23(0.97-1.56)$ if present $(n=93)$; $2.79(1.00-7.81)$ if absent $(n=27)$; interaction $p=0.287)$. In PETROS, the association of $\triangle$ RIPK3 with ARDS was similar when patients were stratified by trauma mechanism (adjusted OR $1.93(1.39-2.69)$ for blunt trauma $(n=$ 137); $1.96(1.16-3.34)$ for penetrating trauma $(n=43)$; interaction $p=0.958$ ). 
Table 1 Baseline patient characteristics of patients in MESSI and PETROS cohorts

\begin{tabular}{|c|c|c|}
\hline & MESSI cohort $(n=120)$ & PETROS cohort $(n=180)$ \\
\hline \multicolumn{3}{|l|}{ Demographics } \\
\hline Age, years & $61(50-68)$ & $41(25-62)$ \\
\hline Male sex & $71(59)$ & $140(78)$ \\
\hline \multicolumn{3}{|l|}{ Race $^{a}$} \\
\hline White & $63(53)$ & $76(42)$ \\
\hline Black & $51(43)$ & $89(49)$ \\
\hline Other $^{b}$ & $6(4)$ & $15(8)$ \\
\hline Body mass index $\left(\mathrm{kg} / \mathrm{m}^{2}\right)$ & $26.5(22.1-32.7)$ & $25.3(22.7-28.5)$ \\
\hline \multicolumn{3}{|l|}{ Medical history } \\
\hline Hypertension $^{a}$ & $70(58)$ & $50(28)$ \\
\hline Diabetes mellitus $^{a}$ & $45(38)$ & $13(7)$ \\
\hline Congestive heart failure $^{a}$ & $21(18)$ & $7(4)$ \\
\hline Chronic kidney disease $\mathrm{e}^{a c}$ & $20(17)$ & $5(3)$ \\
\hline Chronic lung disease $^{a}$ & $15(13)$ & $0(0)$ \\
\hline Chronic alcohol abuse $e^{a d}$ & $15(13)$ & $10(6)$ \\
\hline \multicolumn{3}{|l|}{ Smoking history ${ }^{a d}$} \\
\hline Never & $56(47)$ & $66(40)$ \\
\hline Former & $27(23)$ & $25(15)$ \\
\hline Current & $19(16)$ & $72(44)$ \\
\hline \multicolumn{3}{|l|}{ Acute injury and illness } \\
\hline Blunt trauma mechanism & N/A & $137(76)$ \\
\hline Injury Severity Score & N/A & $25(19-30)$ \\
\hline Operation prior to ICU admission & N/A & $74(41)$ \\
\hline APACHE ॥ & $29(21-37)$ & $17(12-24)$ \\
\hline Shock prior to ICU admission ${ }^{a e}$ & $93(78)$ & $89(50)$ \\
\hline Crystalloid, liters ${ }^{\text {adf }}$ & $3.5(2.0-6.9)$ & $2.5(1.2-4.0)$ \\
\hline Pulmonary source of sepsis & $51(43)$ & N/A \\
\hline \multicolumn{3}{|l|}{ Transfusions (days $0+1)^{9}$} \\
\hline Received RBC transfusion & $31(26)$ & $108(60)$ \\
\hline Number of units & $2(1-2)$ & $5(3-9)$ \\
\hline Received FFP transfusion & $16(13)$ & $67(37)$ \\
\hline Number of units & $3(2-4)$ & $4(2-6)$ \\
\hline Received platelet transfusion & $18(15)$ & $62(34)$ \\
\hline Number of doses ${ }^{h}$ & $2(1-3)$ & $2(1-2)$ \\
\hline \multicolumn{3}{|l|}{ Outcomes } \\
\hline ARDS & $44(37)$ & $37(21)$ \\
\hline $\mathrm{AKI}^{i}$ & $41(37)$ & $53(30)$ \\
\hline 30-day mortality & $50(42)$ & $17(9)$ \\
\hline
\end{tabular}

Data are shown as $n$ (\%) for categorical variables and median (interquartile range) for continuous variables. Definition of abbreviations: ARDS acute respiratory distress syndrome, SBP systolic blood pressure, ED emergency department, ICU intensive care unit, $R B C$ red blood cell, FFP fresh frozen plasma. ${ }^{a}$ Missing data for PETROS cohort: race $(n=4)$; hypertension [4]; diabetes [3]; coronary heart disease [3]; congestive heart failure [2]; chronic kidney disease [1]; chronic lung disease [1]; chronic alcohol abuse [5]; smoking history [17]; shock prior to ICU admission [1]; crystalloid [4]. ${ }^{b}$ In PETROS cohort, Asian $(n=9)$, North American Indian/ Alaskan Native ( $n=2)$, and Unknown $(n=2)$; in MESSI cohort, Asian $(n=1)$ and unknown $(n=5)$. Includes patients with end-stage renal disease (MESSI $n=9$, PETROS $n=1$ ). ${ }^{d}$ Missing data for MESSI cohort: chronic alcohol abuse $(n=19)$; smoking history [18]; crystalloid [2]. ${ }^{e}$ Shock defined as need for vasopressors or mean arterial pressure $<65 \mathrm{mmHg}$ (MESSI) or systolic arterial pressure $<90 \mathrm{mmHg}$ (PETROS). ${ }^{f}$ Administered during the first $24 \mathrm{~h}$ after ED presentation (MESSI) or prior to ICU arrival (PETROS). ${ }^{9}$ The calendar day of and the day after presentation. ${ }^{h}$ Each platelet dose at our institution is roughly equivalent to 4 single-donor platelet units or 6 pooled platelet units. 'AKI numbers exclude patients with end-stage renal disease 
Table 2 Unadjusted associations of plasma RIPK3 concentrations with organ dysfunction and death

A. MESSI Cohort

\begin{tabular}{l|c|c|c|c|c|c} 
& $\begin{array}{c}\text { RIPK3 }(\mathbf{p g} / \mathbf{m l}), \\
\text { presentation }\end{array}$ & $\boldsymbol{p}$ & $\begin{array}{c}\text { RIPK3 }(\mathbf{p g} / \mathbf{m l}), \\
\mathbf{4 8 h}\end{array}$ & $\boldsymbol{p}$ & $\begin{array}{c}\Delta \text { RIPK3 }(\mathbf{p g} / \mathbf{m l}), \\
\text { presentation to 48h }\end{array}$ & $\boldsymbol{p}$ \\
\hline ARDS & $131(56-257)$ & 0.929 & $200(77-494)$ & 0.054 & $42(0-200)$ & 0.006 \\
No ARDS & $110(36-320)$ & & $98(22-399)$ & & $0(-61-64)$ & \\
AKI & $122(56-350)$ & 0.180 & $291(82-534)$ & 0.005 & $50(0-293)$ & 0.006 \\
No AKI & $111(36-233)$ & & $100(16-258)$ & & $0(-59-58)$ & \\
Dead at 30 days & $150(49-413)$ & 0.068 & $256(77-666)$ & 0.002 & $29(-16-290)$ & 0.049 \\
Alive at 30 days & $110(34-235)$ & & $97(22-276)$ & & $0(-60-72)$ &
\end{tabular}

\section{B. PETROS Cohort}

\begin{tabular}{|c|c|c|c|c|c|c|}
\hline & $\begin{array}{l}\text { RIPK3 }(\mathrm{pg} / \mathrm{ml}) \\
\text { presentation }\end{array}$ & $p$ & $\begin{array}{c}\text { RIPK3 }(\mathrm{pg} / \mathrm{ml}) \text {, } \\
48 \mathrm{~h}\end{array}$ & $p$ & $\begin{array}{c}\Delta R I P K 3(\mathrm{pg} / \mathrm{ml}), \\
\text { presentation to } 48 \mathrm{~h}\end{array}$ & $p$ \\
\hline ARDS & $16(16-29)$ & 0.548 & $195(129-401)$ & $<0.001$ & $164(100-316)$ & $<0.001$ \\
\hline No ARDS & $16(16-49)$ & & $104(30-186)$ & & $47(0-130)$ & \\
\hline \multirow[t]{2}{*}{ AKI } & $16(16-37)$ & 0.183 & $162(75-343)$ & 0.004 & $134(33-295)$ & $<0.001$ \\
\hline & $\begin{array}{l}\text { RIPK3 }(\mathrm{pg} / \mathrm{ml}) \\
\text { presentation }\end{array}$ & $p$ & $\begin{array}{c}\text { RIPK3 }(\mathrm{pg} / \mathrm{ml}) \text {, } \\
48 \mathrm{~h}\end{array}$ & $p$ & $\begin{array}{c}\Delta R I P K 3(\mathrm{pg} / \mathrm{ml}), \\
\text { presentation to } 48 \mathrm{~h}\end{array}$ & $p$ \\
\hline No AKI & $16(16-64)$ & & $110(31-187)$ & & $46(0-130)$ & \\
\hline Dead at 30 days & $16(16-81)$ & 0.469 & 233 (116-394) & 0.006 & $152(43-231)$ & 0.022 \\
\hline Alive at 30 days & $16(16-47)$ & & $122(34-195)$ & & $56(0-146)$ & \\
\hline
\end{tabular}

All $p$ values are from comparisons using the Wilcoxon rank-sum test. Analyses involving AKI exclude patients with end-stage renal disease (MESSI cohort $n=9$; PETROS cohort $n=1$ ). Definition of abbreviations: ARDS acute respiratory distress syndrome, AKI acute kidney injury, RIPK3 receptor interacting protein kinase-3

We further analyzed whether $\triangle$ RIPK3 elevations might precede ARDS by comparing patients without ARDS to only those who developed ARDS after the first $48 \mathrm{~h}$ (the second time point of RIPK3 testing). In PETROS, 18/37 (48.6\%) ARDS patients developed ARDS after $48 \mathrm{~h}$. These patients had significantly higher $\triangle$ RIPK3 levels than those without ARDS (median 163.7 $\mathrm{pg} / \mathrm{ml}$ vs. $48.9 \mathrm{pg} / \mathrm{ml}$, respectively, $p<0.001$, Additional file 2: Figure S1). In MESSI, there were too few patients who developed ARDS after $48 \mathrm{~h}(n=5)$ to conduct a similar subgroup analysis.

Because phenytoin may inhibit necroptosis [36], we examined the impact of phenytoin use on $\triangle$ RIPK3 and its relationship with ARDS. In PETROS, the 27/180 (15\%) patients who received phenytoin within the first $48 \mathrm{~h}$ had lower median $\triangle$ RIPK3 levels than those who did not (36.7 (IQR 0-104.8) pg/ml vs. $68.5(0-178.5)$ $\mathrm{pg} / \mathrm{ml}$, respectively), though the difference was not statistically significant $(p=0.071)$. Adding phenytoin to the primary multivariable model, however, minimally changed the association of $\triangle$ RIPK3 and ARDS (OR 1.83 (95\% CI 1.35-2.49), $p<0.001$ ). Only two MESSI patients received phenytoin in the first $48 \mathrm{~h}$, precluding similar analyses in this cohort.

Plasma and lung RIPK3 in mouse model

It has previously been reported that lung RIPK3 and MLKL are elevated following intratracheal LPS administration [10]. We asked whether lung RIPK3 and MLKL expression are elevated following systemic LPS, which induces a systemic inflammatory state relevant to human sepsis and trauma, and whether plasma RIPK3 shows a concomitant rise. Both lung RIPK3 expression (Fig. 2a, b) and plasma RIPK3 concentration (Fig. 2c) were 
Table 3 Multivariable logistic regression models of the $\triangle$ RIPK3-ARDS association adjusted for pre-specified confounders
A. MESSI Cohort.

\begin{tabular}{l|c|c} 
Covariate & Odds ratio (95\% Cl) & $\boldsymbol{p}$ \\
\hline$\triangle$ RIPK3 (per 1/2 SD) & $1.30(1.03-1.63)$ & 0.027 \\
Age & $0.98(0.96-1.02)$ & 0.393 \\
RBCs transfused, presentation day (per unit) & $1.48(1.03-2.12)$ & 0.035 \\
Lung source of sepsis & $7.15(2.75-18.56)$ & $<0.001$ \\
Shock at presentation $^{a}$ & $3.65(1.18-11.34)$ & 0.026 \\
\hline
\end{tabular}

\section{B. PETROS Cohort.}

\begin{tabular}{l|c|c} 
Covariate & Odds ratio $(95 \%$ Cl) & $p$ \\
\hline$\Delta$ RIPK3 (per 1/2 SD) & $1.83(1.35-2.48)$ & $<0.001$ \\
RBCs transfused, first 6h (per unit) & $1.11(0.97-1.27)$ & 0.136 \\
Blunt trauma mechanism & $4.17(1.11-15.64)$ & 0.034 \\
\hline Injury severity score (per 5 points) & $1.12(0.91-1.38)$ & 0.288 \\
\hline
\end{tabular}

For A. and B.: $\triangle$ RIPK3 remains significantly associated with ARDS after adjustment for pre-specified confounders. The odds ratio corresponds to the adjusted association of each covariate with ARDS. ${ }^{a}$ Shock defined as need for vasopressors or mean arterial pressure $<65 \mathrm{mmHg}$. Definition of abbreviations: ARDS acute respiratory distress syndrome, RIPK3 receptor interacting protein kinase-3, SD standard deviation, RBCs red blood cells

significantly elevated following LPS or LPS in the presence of the pan-caspase inhibitor ZVAD-FMK when compared with PBS or ZVAD-FMK alone. There was a positive correlation between lung and plasma RIPK3 (Fig. 2d), though this correlation did not reach statistical significance (Spearman's $\rho=0.55, p=0.102$ ). We did not observe increased lung MLKL or phosphorylated MLKL expression following systemic LPS (Additional file 2: Figure S2).

\section{Secondary analyses}

In MESSI, $\triangle$ RIPK3 and 48-h RIPK3 were significantly higher in patients who developed AKI than those who did not (Table 2). This association was also present in 180 PETROS patients, which included 80 patients in whom we previously reported a RIPK3-AKI association [19]. These associations were independent of prespecified confounders (Additional file 1: Table S1 and Additional file 2: Figure S3). In both cohorts, those with both AKI and ARDS had the highest $\triangle$ RIPK3 levels, though patients with AKI or ARDS only still had significantly higher $\triangle R I P K 3$ than those with neither (Additional file 2: Figure S4). The associations of $\triangle$ RIPK3 with either ARDS or AKI largely remained significant when adjusted for AKI or ARDS, respectively (Additional file 1: Table S2).

$\triangle$ RIPK3 plasma concentration was also associated with mortality in both cohorts (Table 2). In MESSI, this association remained significant after adjustment for presence of shock at presentation and other confounders (adjusted OR 1.27, 95\% CI 1.03-1.57, $p=0.028$; Additional file 1: Table S3).

Additional file 1 Table S4 shows the association of patient characteristics with $\triangle \mathrm{RIPK} 3$ in each cohort. APACHE II score was strongly associated with $\triangle \mathrm{RIPK} 3$ in both sepsis and trauma patients. $\triangle \mathrm{RIPK} 3$ also increased with greater numbers of blood products, though this association was most notable in PETROS, in which transfusions were far more common. In PETROS, younger age, non-Caucasian race, penetrating trauma, shock prior to ICU admission, need for emergent operation, and increased crystalloid resuscitation were also associated with higher $\triangle$ RIPK3. Underweight and obese MESSI patients had higher $\triangle$ RIPK3 than normal and overweight patients, though these findings were not statistically significant $(p=0.073)$. As noted, the majority of PETROS patients had presentation RIPK3 levels below the limit of 


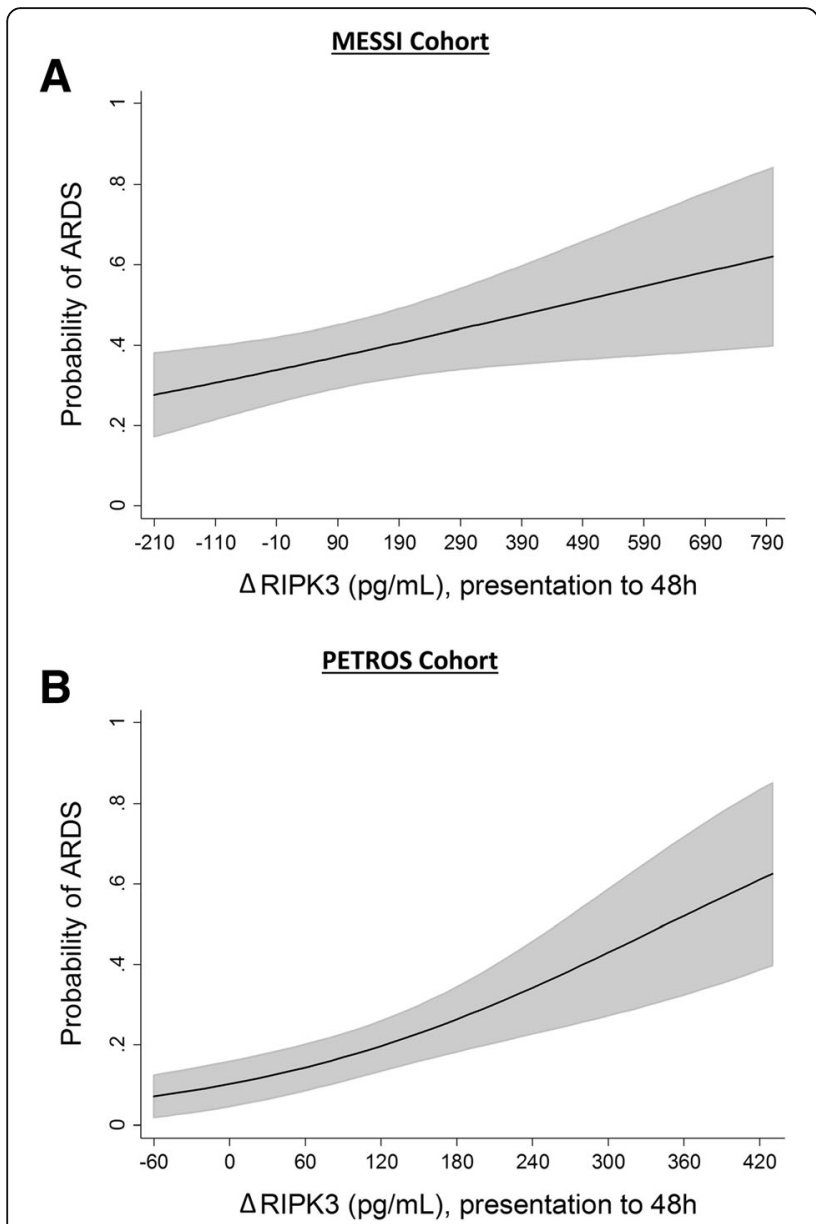

Fig. 1 Adjusted probability of acute respiratory distress syndrome (ARDS) across the range of $\triangle$ RIPK3 (change from presentation to 48 h) in each cohort. Estimated probabilities (line) with 95\% confidence intervals (gray shading) determined using post-estimation marginal analysis after multivariable logistic regression modeling. a MESSI cohort, probabilities adjusted for age, red blood cell transfusions on day of presentation, lung source of sepsis, and shock at presentation. $\mathbf{b}$ PETROS cohort, probabilities adjusted for red blood cell transfusions in the first $6 \mathrm{~h}$, trauma mechanism, and injury severity score

detection; only congestive heart failure and blunt trauma mechanism were associated with higher RIPK3 levels at this time point (Additional file 1: Table S5). In MESSI, however, younger age, chronic alcohol abuse, higher APACHE II score, and higher volume of crystalloid resuscitation were associated with presentation RIPK3 concentrations. In addition, median presentation RIPK3 was higher in patients who died by day 30 , though this difference was not statistically significant $(p=0.068)$.

\section{Discussion}

In this study, we demonstrated that the change in plasma RIPK3 concentration from presentation to $48 \mathrm{~h}$ was independently associated with ARDS in two major at-risk populations, sepsis and trauma and that both lung RIPK3 expression and plasma RIPK3 concentrations rose significantly in mice treated with systemic LPS alone or when given with an inhibitor of apoptosis. Despite a wealth of recent animal and tissue studies of necroptosis showing its potential relevance to multiple human syndromes including ARDS $[9,10,15,16]$, data in human populations remain limited [13, 19-21, 37]. Our study provides the largest analysis to date of RIPK3 in ARDS, takes steps toward understanding the role of plasma RIPK3 as a marker of lung injury, and strengthens prior findings that plasma RIPK 3 was associated with AKI and mortality. In the context of existing preclinical data $[10,13]$, these findings collectively suggest that necroptosis and other RIPK3-regulated pathways may be mechanistically important in ARDS and other acute organ dysfunction syndromes.

Since its description in 2009 [38], RIPK3-mediated necroptosis has emerged as a key mechanism in preclinical models of acute lung and renal injury [10, 13, 15]. The characteristic release of tissue-injurious DAMPs during necroptosis makes it of great interest as a driver, and therefore potential therapeutic target, of acute organ injury [13, 34, 39-41]. In sepsis patients, Davenport et al. identified increased gene expression of RIPK3 and MLKL in circulating leukocytes as part of a molecular response subtype characterized by a two- to threefold mortality increase [42]. There are now reports of plasma RIPK 3 associated with mortality [13, 19, 21], AKI [19, 37], and mechanical ventilation [20]. Studies on RIPK3 in ARDS, however, are limited, the largest being a subgroup analysis that included 24 patients with ARDS [20]. In cohorts with over three times that number of ARDS cases, we now show a convincing association of plasma RIPK3 with ARDS independent of relevant confounders. We also add novel findings about the time course of the RIPK3-ARDS association. While the rise in plasma RIPK3 over the first $48 \mathrm{~h}$ was clearly able to distinguish ARDS from non-ARDS cases, there was no signal that RIPK3 on presentation to the ED or trauma bay could predict ARDS, with similar findings for AKI and mortality. These results have potential implications for clinical utility: by $48 \mathrm{~h}$, when ARDS is often already manifest, this biomarker may be most helpful to identify a subgroup with RIPK3 activation for possible targeted treatment. In fact, RIPK3 inhibitors have already shown protection against tissue injury in preclinical studies [35, 43], and efforts to translate these findings into effective therapies may be aided by understanding patient groups most likely to respond. For consideration of RIPK3 as an ARDS prediction or prevention tool, however, studies of serial early measurements would be needed to determine how rapidly after presentation the RIPK3-ARDS association becomes evident. 
A

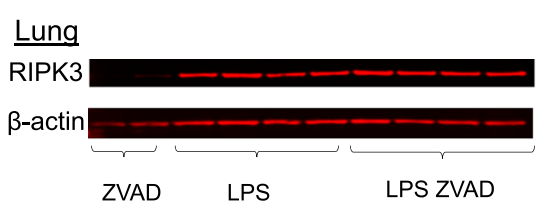

B

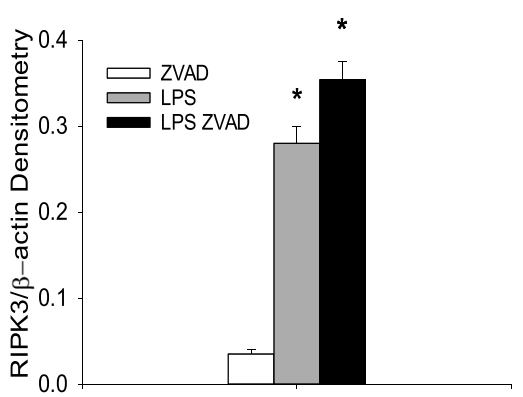

C

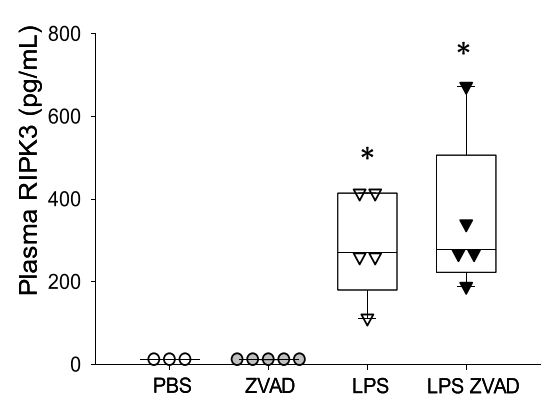

D

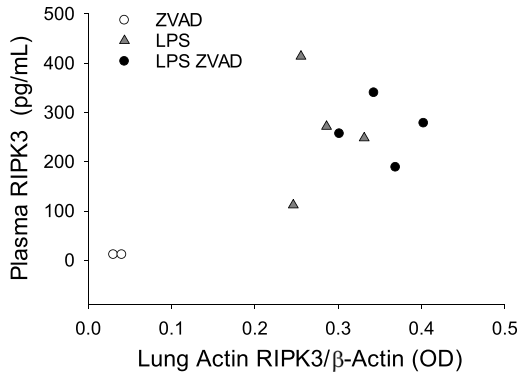

Fig. 2 Lung and plasma RIPK3 are elevated following LPS and LPS-ZVAD treatment. a Whole lung homogenate of mice $4 \mathrm{~h}$ following LPS, LPSZVAD treatment; $n=2$ studies (5-8 mice/group), immunoblot from one study is shown. $\mathbf{b}$ Densitometry of whole lung homogenate, ${ }^{*} p=0.016$ LPS vs. ZVAD, $p=0.005$ LPS-ZVAD vs. ZVAD. c Plasma RIPK3, ${ }^{*} p=0.002$ LPS vs. PBS or ZVAD, $p=0.002$ LPS-ZVAD vs. PBS or ZVAD. d Correlation of lung tissue and plasma RIPK3 (Spearman's $\rho=0.55, p=0.102$ )

For any such pathway-targeted applications, though, it is important to know to what degree plasma RIPK3 reflects underlying lung injury. We previously demonstrated that human vascular endothelial cells undergoing necroptosis release RIPK3 [13], but it remained unclear if in vivo circulating RIPK3 levels reflected RIPK3 expression in lung tissue. Our current finding that murine lung and plasma RIPK3 rose substantially and concomitantly in response to systemic LPS and LPS-ZVAD suggests that the RIPK3-ARDS association in sepsis and trauma patients could reflect increased expression and release of RIPK3 from injured lung tissue, injury that is not explained by apoptosis. While necroptotic cell death is one explanation for these findings, other RIPK3-dependent pathways may be involved: Lawlor et al. have shown that RIPK3 can promote inflammasome activation independent of MLKL and necroptosis [44]. Notably, we found that pMLKL, an intracellular mediator of necroptosis downstream of RIPK3, did not increase after LPS or LPSZVAD. These findings are also consistent with those of Siempos et al. in which RIPK3-deficient mice were protected from ventilator-induced lung injury while MLKL-deficient mice were not [20]. Further studies, potentially including testing of plasma pMLKL and other key cell death pathway mediators that may be involved in the RIPK3-ARDS association, are important if therapies targeting programmed necrosis are to be considered for acute lung injury.

Our study expands on smaller reports that plasma RIPK3 is associated with AKI in sepsis and trauma patients $[19,37]$. We now demonstrate a RIPK3-AKI association robust to adjustment for relevant confounders and independent of ARDS. This lends further clinical relevance to multiple preclinical studies showing the importance of RIPK3 and necroptosis in acute renal injury $[8,12,15,37,45]$. It is highly plausible that programmed necrosis in the kidneys, as well as the lungs and other organs, results in elevated circulating RIPK3 levels in sepsis and trauma patients. It is also possible that circulating RIPK3 is itself a causal factor in multiple organ dysfunction, similar to well-established DAMPs like cellfree DNA. In either case, RIPK3 may identify a process of necroinflammation in which the release of a diverse groups of DAMPs by necrotic cells serve to propagate and sustain the inflammatory response [46]. For example, we previously found that the DAMP highmobility group box 1 protein (HMGB1) released following transfusion-induced necroptosis primes mice to subsequent lung injury [13], and others showed that cigarette smoke-induced necroptosis and DAMP release increase airway inflammation [47]. The complexity of how necroptosis and other regulated necrosis pathways result in and interact with release of myriad DAMPs in vivo to 
promote inflammation, tissue injury, and multiple organ dysfunction remains inadequately understood. Clinically relevant animal models of sepsis and trauma may be best suited to clarify these knowledge gaps.

There are limited existing data on patient characteristics associated with RIPK3 levels [19, 20]. We found that severity of illness measures tracked with plasma $\triangle$ RIPK 3 in both sepsis and trauma. In MESSI but not PETROS, this was true for presentation RIPK3 as well, possibly reflecting a greater delay from initial insult to ED presentation in sepsis patients, allowing more time for circulating RIPK3 to rise. In PETROS, variables including race, penetrating trauma, shock, crystalloid volume, and blood product transfusions were all significantly associated with $\triangle$ RIPK3. We have previously shown that RBCs can induce RIPK3 release from lung endothelial cells [13]. Transfused patients are also at increased risk of ARDS [30]. If RIPK3 proves to be a causal link, targeting RIPK3 pathways could be considered to reduce ARDS rates among the substantial number of trauma patients requiring transfusions.

Our study has several limitations. First, we did not have plasma samples at time points between presentation and $48 \mathrm{~h}$. The kinetics of plasma RIPK 3 in the early hours of sepsis and trauma remain unclear, as does the ability of RIPK3 at such time points to predict subsequent ARDS. While we showed in trauma patients that $\triangle$ RIPK3 was associated with ARDS developing after 48 $h$, future studies with serial RIPK3 measurements may provide more granular detail of the time-varying relationship of RIPK3 and ARDS during early critical illness to allow for more robust causal inference. Second, while our study is the largest analysis to date of RIPK3 and ARDS, our cohort sizes did not allow adjustment for all possible confounders without potentially overfitting the multivariable models. Third, while the significant rise in murine plasma RIPK3 concentrations in response to LPS and LPS-ZVAD mirrored that seen in lung tissue, larger studies would be needed to firmly establish a tight correlation of plasma and lung RIPK3 concentrations. Studies testing RIPK3 expression in other organs may help to determine whether plasma RIPK3 also reflects extra-pulmonary tissue expression. Fourth, the specificity of RIPK3 as a marker of necroptosis in the lung or other organs remains unknown in human populations. Future studies could include using tissue or fluid obtained from affected organs, such as bronchoalveolar lavage fluid or urine, to validate plasma RIPK3 as a non-invasive marker of necroptosis or other RIPK3-related pathways. Finally, whether clinically available tests reflecting cell death such as lactate dehydrogenase strongly correlate with RIPK3, and therefore could be used as surrogates, remains unclear but could be tested in future studies.

\section{Conclusions}

We demonstrated a significant association of plasma $\triangle$ RIPK3 with ARDS in critically ill sepsis and trauma patients and showed that both lung and plasma RIPK3 increased rapidly in mice injected with LPS-ZVAD. These findings extend prior preclinical studies and suggest that necroptosis and other RIPK3-dependent processes may be important mechanisms underlying ARDS in these two at-risk populations. Ongoing studies of this marker may prove useful in identifying novel molecular pathways to target for ARDS prevention and treatment.

\section{Additional files}

Additional file 1: Methods, STROBE statement, and supplemental
tables. (DOCX $127 \mathrm{~kb}$ )

Additional file 2: Figure S1. Change in plasma RIPK3 concentration from presentation to $48 \mathrm{~h}$ by Acute Respiratory Distress Syndrome (ARDS) status excluding patients who met ARDS criteria $\leq 48 \mathrm{~h}$ after presentation among patients in the PETROS (trauma) cohort (No ARDS $n=143$, ARDS $n=18$ meeting criteria 55-140 h after presentation). Gray boxes represent interquartile range, with median designated by central line. Figure S2. Levels of mixed lineage kinase domain-like protein (MLKL) and phosphorylated MLKL (pMLKL) in whole lung homogenate are not significantly different between mice treated with ZVAD, LPS, or LPS ZVAD. A. Immunoblot of whole lung homogenate showing similar $M L K L$ and $\mathrm{pMLKL}$ regardless of treatment. B. Densitometry of whole lung homogenate. All comparisons between treatment groups are non-significant. Figure S3. Adjusted probability of acute kidney injury (AKI) across range of $\Delta$ receptor interacting protein kinase-3 (RIPK3) levels (RIPK3 change from presentation to $48 \mathrm{~h}$ ) in each cohort. Estimated probabilities (line) with 95\% confidence intervals (gray shading) determined using post-estimation marginal analysis after multivariable logistic regression modeling. A. MESSI cohort. Probabilities adjusted for age, red blood cell transfusions on the day of presentation, chronic kidney disease, diabetes mellitus, and shock at presentation. B. PETROS cohort. Probabilities adjusted for red blood cell transfusions in the first $6 \mathrm{~h}$ after presentation, trauma mechanism, abdominal injury severity, and shock prior to ICU admission. Figure S4. Plasma levels of $\Delta$ receptor interacting protein kinase-3 ( $\triangle$ RIPK3, change from presentation to $48 \mathrm{~h}$ ) by acute kidney injury (AKI) and acute respiratory distress syndrome (ARDS) category in human cohorts. Shaded portions of box plots show median concentrations (central line) and 25th and 75th percentiles (bottom and top lines of box). Brackets above the $p$ values denote the two organ dysfunction categories being compared (Wilcoxon rank-sum test). A. MESSI cohort. Patients with AKI only, ARDS only, or AKI + ARDS had higher $\triangle R I P K 3$ than those with neither AKI nor ARDS. B. PETROS cohort. Patients with AKI + ARDS had higher $\triangle$ RIPK3 than those with AKI or ARDS only, groups which in turn had higher $\triangle$ RIPK3 than patients with neither. (ZIP $1021 \mathrm{~kb})$

\footnotetext{
Abbreviations

AKI: Acute kidney injury; AKIN: Acute Kidney Injury Network; ARDS: Acute respiratory distress syndrome; DAMP: Damage-associated molecular pattern; ELISA: Enzyme-linked immunosorbent assay; HMGB1: High-mobility group box 1 protein; ISS: Injury Severity Score; LPS: Lipopolysaccharide; MESSI: Molecular Epidemiology of SepsiS in the ICU cohort; MLKL: Mixed lineage kinase domain-like protein; PETROS: PEnn TRauma acute Organ dysfunction Study; pMLKL: Phosphorylated MLKL; RBC: Red blood cell; RIPK3: Receptor interacting serine/threonine-protein kinase-3; RRT: Renal replacement therapy; SDS-PAGE: Sodium dodecyl sulfate polyacrylamide gel electrophoresis; $\triangle$ RIPK3: Change in RIPK3 concentration from presentation through approximately $48 \mathrm{~h}$ later
} 


\section{Acknowledgements}

The authors would like to acknowledge the faculty and staff at the Penn Trauma Center, the medical ICU at the Hospital of the University of Pennsylvania, and the clinical laboratories at the Hospital of the University of Pennsylvania and Penn Presbyterian Medical Centers for their assistance in the conduct of this study.

\section{Authors' contributions}

MGSS, JPR, NJM, and NSM designed the study. PXZ, MJH, and NSM conducted animal experiments. MGSS, JPR, CMF, CAGI, BJA, DNH, PNL, JDC, and NJM conducted the clinical study. MGSS, JPR, CMF, CAGI, DF, and NJM collected data for the clinical study. MGSS, JPR, WY, and NSM conducted all analyses and drafted the manuscript. MGSS and JPR contributed equally to the study. All authors critically revised and approved the manuscript.

\section{Funding}

This study was funded by NIH grants P50HL060290, P01HL079063, K24HL115354, K08HL131995, R01DK111638, K08HL098362, K23HL125723, K23HL140482, and the Department of Defense-W81XWH-15-1-0363. The funders had no role in study design, data collection and analysis, decision to publish, or preparation of the manuscript.

\section{Availability of data and materials}

The authors will furnish deidentified copies of the analytic datasets used upon reasonable request, pending permission from the University of Pennsylvania Institutional Review Board.

\section{Ethics approval and consent to participate}

The MESSI and PETROS prospective cohorts were approved by the University of Pennsylvania Institutional Review Board. Informed consent was obtained from patients enrolled in MESSI. PETROS was approved by the IRB with a waiver of informed consent.

\section{Consent for publication}

Not applicable.

\section{Competing interests}

Dr. Meyer reports institutional funding from GlaxoSmithKline and served on the advisory board for SOBI, Inc. Dr. Christie reports institutional funding from GlaxoSmithKline. Dr. Mangalmurti reports receipt of RIP1KD mice and RIP1K inhibitors from GlaxoSmithKline for murine studies to determine the role of RIP1K dependent necroptosis in transfusion mediated lung injury. The remaining authors declare that they have no competing interests.

\section{Author details}

'Pulmonary, Allergy, and Critical Care Division, Perelman School of Medicine, University of Pennsylvania, 5039 W Gates Building, 3600 Spruce Street, Philadelphia, PA 19104, USA. ${ }^{2}$ Center for Clinical Epidemiology and Biostatistics, Perelman School of Medicine, University of Pennsylvania, Pennsylvania, USA. ${ }^{3}$ Center for Translational Lung Biology, Perelman School of Medicine, University of Pennsylvania, Pennsylvania, USA. ${ }^{4}$ Division of Traumatology, Surgical Critical Care, and Emergency Surgery, Perelman School of Medicine, University of Pennsylvania, Pennsylvania, USA.

\section{Received: 20 December 2018 Accepted: 19 May 2019}

\section{Published online: 28 June 2019}

\section{References}

1. Rubenfeld GD, Caldwell E, Peabody E, Weaver J, Martin DP, Neff M, et al. Incidence and outcomes of acute lung injury. N Engl J Med. 2005; 353(16):1685-93.

2. Shah CV, Localio AR, Lanken PN, Kahn JM, Bellamy S, Gallop R, et al. The impact of development of acute lung injury on hospital mortality in critically ill trauma patients. Crit Care Med. 2008;36(8):2309-15.

3. Reilly JP, Anderson BJ, Mangalmurti NS, Nguyen TD, Holena DN, Wu Q, et al. The ABO histo-blood group and AKI in critically ill patients with trauma or sepsis. Clin J Am Soc Nephrol. 2015;10(11):1911-20

4. Herridge MS, Tansey CM, Matté A, Tomlinson G, Diaz-Granados N, Cooper A, et al. Functional disability 5 years after acute respiratory distress syndrome. N Engl J Med. 2011;364(14):1293-304.
5. Brower RG, Matthay MA, Morris A, Schoenfeld D, Thompson BT, Wheeler A. Ventilation with lower tidal volumes as compared with traditional tidal volumes for acute lung injury and the acute respiratory distress syndrome. N Engl J Med. 2000;342(18):1301-8.

6. Guérin C, Reignier J, Richard JC, Beuret P, Gacouin A, Boulain T, et al. Prone positioning in severe acute respiratory distress syndrome. N Engl J Med. 2013;368(23):2159-68.

7. Thompson BT, Chambers RC, Liu KD. Acute respiratory distress syndrome. N Engl J Med. 2017;377(6):562-72.

8. Linkermann A, Brasen JH, De Zen F, Weinlich R, Schwendener RA, Green DR et al. Dichotomy between RIP1- and RIP3-mediated necroptosis in tumor necrosis factor-alpha-induced shock. Mol Med. 2012;18:577-86.

9. Linkermann A, Green DR. Necroptosis. N Engl J Med. 2014;370(5):455-65.

10. Wang L, Wang T, Li H, Liu Q, Zhang Z, Xie W, et al. Receptor interacting protein 3-mediated necroptosis promotes lipopolysaccharide-induced inflammation and acute respiratory distress syndrome in mice. PLoS One. 2016;11(5):e0155723.

11. Louhimo JM, Steer ML, Perides G. Necroptosis is an important severity determinant and potential therapeutic target in experimental severe pancreatitis. Cell Mol Gastroenterol Hepatol. 2016;2(4):519-35.

12. Linkermann A, Bräsen JH, Himmerkus N, Liu S, Huber TB, Kunzendorf U, et al. Rip1 (receptor-interacting protein kinase 1) mediates necroptosis and contributes to renal ischemia/reperfusion injury. Kidney Int. 2012;81(8):751-61.

13. Qing DY, Conegliano D, Shashaty MG, Seo J, Reilly JP, Worthen GS, et al. Red blood cells induce necroptosis of lung endothelial cells and increase susceptibility to lung inflammation. Am J Respir Crit Care Med. 2014;190(11):1243-54.

14. Vandenabeele P, Galluzzi L, Vanden Berghe T, Kroemer G. Molecular mechanisms of necroptosis: an ordered cellular explosion. Nat Rev Mol Cell Biol. 2010;11(10):700-14.

15. Linkermann A, Chen G, Dong G, Kunzendorf U, Krautwald S, Dong Z. Regulated cell death in AKI. J Am Soc Nephrol. 2014;25(12):2689-701.

16. Moreno-Gonzalez G, Vandenabeele P, Krysko DV. Necroptosis: a novel cell death modality and its potential relevance for critical care medicine. Am J Respir Crit Care Med. 2016:194(4):415-28.

17. Kaczmarek A, Vandenabeele P, Krysko DV. Necroptosis: the release of damage-associated molecular patterns and its physiological relevance. Immunity. 2013;38(2):209-23.

18. Pasparakis $\mathrm{M}$, Vandenabeele $\mathrm{P}$. Necroptosis and its role in inflammation. Nature. 2015;517(7534):311-20.

19. Shashaty MG, Reilly JP, Sims CA, Holena DN, Oing D, Forker CM, et al. Plasma levels of receptor interacting protein Kinase-3 (RIP3), an essential mediator of necroptosis, are associated with acute kidney injury in critically ill trauma patients. Shock. 2016;46(2):139-43.

20. Siempos II, Ma KC, Imamura M, Baron RM, Fredenburgh LE, Huh J-W, et al. RIPK3 mediates pathogenesis of experimental ventilator-induced lung injury JCl Insight. 2018;3(9):e97102.

21. Ma KC, Schenck EJ, Siempos II, Cloonan SM, Finkelzstein EJ, Pabon MA, et al. Circulating RIPK3 levels are associated with mortality and organ failure during critical illness. JCl Insight. 2018;3(13):e99692.

22. Palakshappa JA, Anderson BJ, Reilly JP, Shashaty MG, Ueno R, Wu Q, et al. Low plasma levels of adiponectin do not explain acute respiratory distress syndrome risk: a prospective cohort study of patients with severe Sepsis. Crit Care. 2016;20(1):71.

23. Reilly JP, Anderson BJ, Hudock KM, Dunn TG, Kazi A, Tommasini A, et al. Neutropenic sepsis is associated with distinct clinical and biological characteristics: a cohort study of severe sepsis. Crit Care. 2016;20(1):222.

24. Shashaty MGS, Kalkan E, Bellamy SL, Reilly JP, Holena DN, Cummins K, et al. Computed tomography-defined abdominal adiposity is associated with acute kidney injury in critically ill trauma patients. Crit Care Med. 2014;42(7):1619-28.

25. Levy MM, Fink MP, Marshall JC, Abraham E, Angus D, Cook D, et al. 2001 SCCM/ESICM/ACCP/ATS/SIS international sepsis definitions conference. Crit Care Med. 2003;31(4):1250-6.

26. Harris PA, Taylor R, Thielke R, Payne J, Gonzalez N, Conde JG. Research electronic data capture (REDCap)--a metadata-driven methodology and workflow process for providing translational research informatics support. J Biomed Inform. 2009:42(2):377-81.

27. Force TADT. Acute respiratory distress syndrome: the Berlin definition. JAMA. 2012:307(23):2526-33.

28. Mehta RL, Kellum JA, Shah SV, Molitoris BA, Ronco C, Warnock DG, et al. Acute kidney injury network: report of an initiative to improve outcomes in acute kidney injury. Crit Care. 2007;11(2). 
29. Peduzzi P, Concato J, Kemper E, Holford TR, Feinstein AR. A simulation study of the number of events per variable in logistic regression analysis. J Clin Epidemiol. 1996;49(12):1373-9.

30. Watkins TR, Nathens AB, Cooke CR, Psaty BM, Maier RV, Cuschieri J, et al. Acute respiratory distress syndrome after trauma: development and validation of a predictive model. Crit Care Med. 2012;40(8):2295-303.

31. Graubard Bl, Korn EL. Predictive margins with survey data. Biometrics. 1999;55(2):652-9.

32. Wu YT, Tan HL, Huang Q, Sun XJ, Zhu X, Shen HM. zVAD-induced necroptosis in $L 929$ cells depends on autocrine production of TNFalpha mediated by the PKC-MAPKs-AP-1 pathway. Cell Death Differ. 2011;18(1):26-37.

33. Zelic M, Roderick JE, O'Donnell JA, Lehman J, Lim SE, Janardhan HP, et al. RIP kinase 1-dependent endothelial necroptosis underlies systemic inflammatory response syndrome. J Clin Invest. 2018;128(5):2064-75.

34. Duprez L, Takahashi N, Van Hauwermeiren F, Vandendriessche B, Goossens $V$, Vanden Berghe T, et al. RIP kinase-dependent necrosis drives lethal systemic inflammatory response syndrome. Immunity. 2011;35(6):908-18.

35. Kaiser WJ, Sridharan H, Huang C, Mandal P, Upton JW, Gough PJ, et al. Tolllike receptor 3-mediated necrosis via TRIF, RIP3, and MLKL. J Biol Chem. 2013;288(43):31268-79.

36. von Massenhausen A, Tonnus W. Phenytoin inhibits necroptosis. Cell Death and Disease. 2018;9(3):359.

37. Sureshbabu A, Patino E, Ma KC, Laursen K, Finkelsztein EJ, Akchurin O, et al. RIPK3 promotes sepsis-induced acute kidney injury via mitochondrial dysfunction. JCI Insight. 2018;3(11):e98411.

38. Cho Y, Challa S, Moquin D, Genga R, Ray TD, Guildford M, et al. Phosphonylationdriven assembly of the RIP1-RIP3 complex regulates programmed necrosis and virus-induced inflammation. Cell. 2009;137(6):1112-23.

39. Sharma A, Matsuo S, Yang WL, Wang Z, Wang P. Receptor-interacting protein kinase 3 deficiency inhibits immune cell infiltration and attenuates organ injury in sepsis. Crit Care. 2014;18(4):R142.

40. Wu J, Huang Z, Ren J, Zhang Z, He P, Li Y, et al. Mlkl knockout mice demonstrate the indispensable role of Mlkl in necroptosis. Cell Res. 2013;23(8):994-1006.

41. Bolognese AC, Yang WL, Hansen LW, Denning NL, Nicastro JM, Coppa GF, et al. Inhibition of necroptosis attenuates lung injury and improves survival in neonatal sepsis. Surgery. 2018:110-16.

42. Davenport EE, Burnham KL, Radhakrishnan J, Humburg P, Hutton P, Mills TC, et al. Genomic landscape of the individual host response and outcomes in sepsis: a prospective cohort study. Lancet Respir Med. 2016;4(4):259-71.

43. Li JX, Feng JM, Wang Y, Li XH, Chen XX, Su Y, et al. The B-Raf(V600E) inhibitor dabrafenib selectively inhibits RIP3 and alleviates acetaminopheninduced liver injury. Cell Death Dis. 2014;5:e1278.

44. Lawlor KE, Khan N, Mildenhall A, Gerlic M, Croker BA, D'Cruz AA, et al. RIPK3 promotes cell death and NLRP3 inflammasome activation in the absence of MLKL. Nat Commun. 2015;6:6282.

45. Linkermann A, Bräsen JH, Darding M, Jin MK, Sanz AB, Heller JO, et al. Two independent pathways of regulated necrosis mediate ischemia-reperfusion injury. Proc Natl Acad Sci U S A. 2013;110(29):12024-9.

46. Sarhan M, Land WG, Tonnus W, Hugo CP, Linkermann A. Origin and consequences of necroinflammation. Physiological Reviews. 2018;98(2):727-80.

47. Pouwels SD, Zijlstra GJ, van der Toorn M, Hesse L, Gras R, Ten Hacken NH, et al. Cigarette smoke-induced necroptosis and DAMP release trigger neutrophilic airway inflammation in mice. Am J Physiol Lung Cell Mol Physiol. 2016;310(4):L377-86.

\section{Publisher's Note}

Springer Nature remains neutral with regard to jurisdictional claims in published maps and institutional affiliations.

Ready to submit your research? Choose BMC and benefit from:

- fast, convenient online submission

- thorough peer review by experienced researchers in your field

- rapid publication on acceptance

- support for research data, including large and complex data types

- gold Open Access which fosters wider collaboration and increased citations

- maximum visibility for your research: over $100 \mathrm{M}$ website views per year

At $\mathrm{BMC}$, research is always in progress.

Learn more biomedcentral.com/submissions 